\title{
Inhibition of pupillary orienting reflex by novelty in conjunction with recognition memory*
}

\author{
RICK M. GARDNER, SUCHOON S. MO \\ and RICHARD BORREGO \\ Southern Colorado State College \\ Pueblo, Colo. 81001
}

When recognition memory was well formed, stimulus novelty not only failed to elicit the orienting reflex in the form of pupillary dilation, but elicited pupillary constriction instead, demonstrating the relation of negative induction in the original sense of Pavlov's theory relating the mechanisms of the pupillary orienting reflex to recognition memory.

In Pavlov's original theory, the mechanism of external inhibition of conditioned reflex is identified as the same mechanism responsible for elicitation of the orienting reflex (Pavlov, 1927, pp. 12-13, 44). Furthermore, an assumption was made by Pavlov that external inhibition is a case of negative induction of conditioned reflex by orienting reflex (Pavlov, 1928, pp. 382). An implication arising from this assumption is that this negative induction is reciprocal in the sense that inhibition of the orienting reflex may be also regarded as a case of negative induction brought about by the elaboration of conditioned reflex. This strict interpretation of Pavlov's original theory as applied to the orienting reflex complements the theory of orienting reflex formulated by Sokolov (1963). According to this theory, as memory is formed (formation of a neuronal model), the agreement between the afferent information and memory would bring about internal inhibition of the orienting reflex. Stimulus novelty, by creating discordance between the afferent information and memory, would bring about elicitation of the orienting reflex. The theory itself does not deal with any possible inductive relation between the mechanisms of the orienting reflex and memory.

It should be noted that demonstration of the orienting reflex to stimulus novelty and subsequent habituation alone cannot deal with the question of reciprocal induction, and Sokolov's theory is insufficient in this sense. For example, let the pupillary dilation to stimulus novelty be a case of orienting reflex and let the task requirement be that of formation of recognition memory. If habituation of the orienting reflex, in this case the pupillary dilation, is due to negative induction, then this recognition memory should be well formed and should be maintained in a state analogous to the state of "concentration" in the terminology of Pavlov's theory. That is, memory in this state should exercise an inhibitory influence on any stimulus effect extraneous

*This study was partially supported by National Science Foundation Research Fund 65-1-746 to Southern Colorado State College. to memory. Consequently, stimulus novelty should not elicit orienting reflex in the form of pupillary dilation. Quite contrarily, stimulus novelty should elicit pupillary constriction. The issue concerning the negative induction of the orienting reflex by memory required demonstration of pupillary constriction to stimulus novelty, since this phenomenon cannot in any way be predicted by Sokolov's theory.

\section{METHOD}

Four undergraduate males served as Ss. Each $\mathrm{S}$ was presented a list of 10 low meaningful CVC items for $3 \mathrm{sec}$ each on a Stowe memory drum. Immediately after presentation of the inspection list, Ss were tested for recognition memory. Each S was given a mimeographed sheet of 20 items including the 10 items presented on the inspection list plus 10 filler items of similar meaningfulness. Meaningfulness was controlled through use of items ranked 6 to 30 on Archer's scale of meaningfulness (Archer, 1960). Each S designated those items on the test list which had been presented previously. This procedure was repeated until all Ss reached a criterion of two perfect trials on the recognition task.

Following successful completion of the recognition task, each $\mathrm{S}$ was seated before an apparatus designed to measure pupil diameter during presentation of visual stimuli. The apparatus consisted of a rectangular box $4 \mathrm{ft}$ long $\mathrm{x} 2 \mathrm{ft}$ wide with a rear-projection screen mounted at one end. The $S$ viewed stimuli flashed on this screen through an eyepiece located at the other end. CVC trigrams were projected through a slide projector onto the rear-projection screen one item at a time for $15 \mathrm{sec}$ each. During presentation, the $S$ viewed the item with his dominant eye with the other eye occluded. An 8-mm camera photographed the eye at a rate of $1 \mathrm{frame} / \mathrm{sec}$ throughout presentation of the 20 items. The apparatus is similar to that described by Hess (1965). Items presented included the 10 items learned on the recognition memory task and 10 filler items of similar meaningfulness. Order of presentation was randomized for each S.

Each $\mathrm{S}$ was instructed to report verbally as soon as possible whether the item presented was on the original inspection list or not. The E recorded the S's response and the latency between stimulus presentation and report.

\section{RESULTS}

Pupil diameter for each $S$ was determined by projecting the filmed image at $5 \mathrm{X}$ magnification and measuring pupil size. Pupil diameters so measured are presented in Fig. 1. Although statistical analysis is not quite feasible due to the nature of the data, differential pupillary reaction to old and new items is clearly demonstrated for all Ss. Although pupillary dilation to old items is not at all clearly demonstrated, pupillary constriction for new items is pronounced. However, since there is no baseline measure of pupil diameter, the terminologies "dilation" and "constriction" are relative with respect to each other. Still, the pupillary reaction to new items is not that of more pronounced dilation as compared to old items.

For each S, the average latency between onset of 


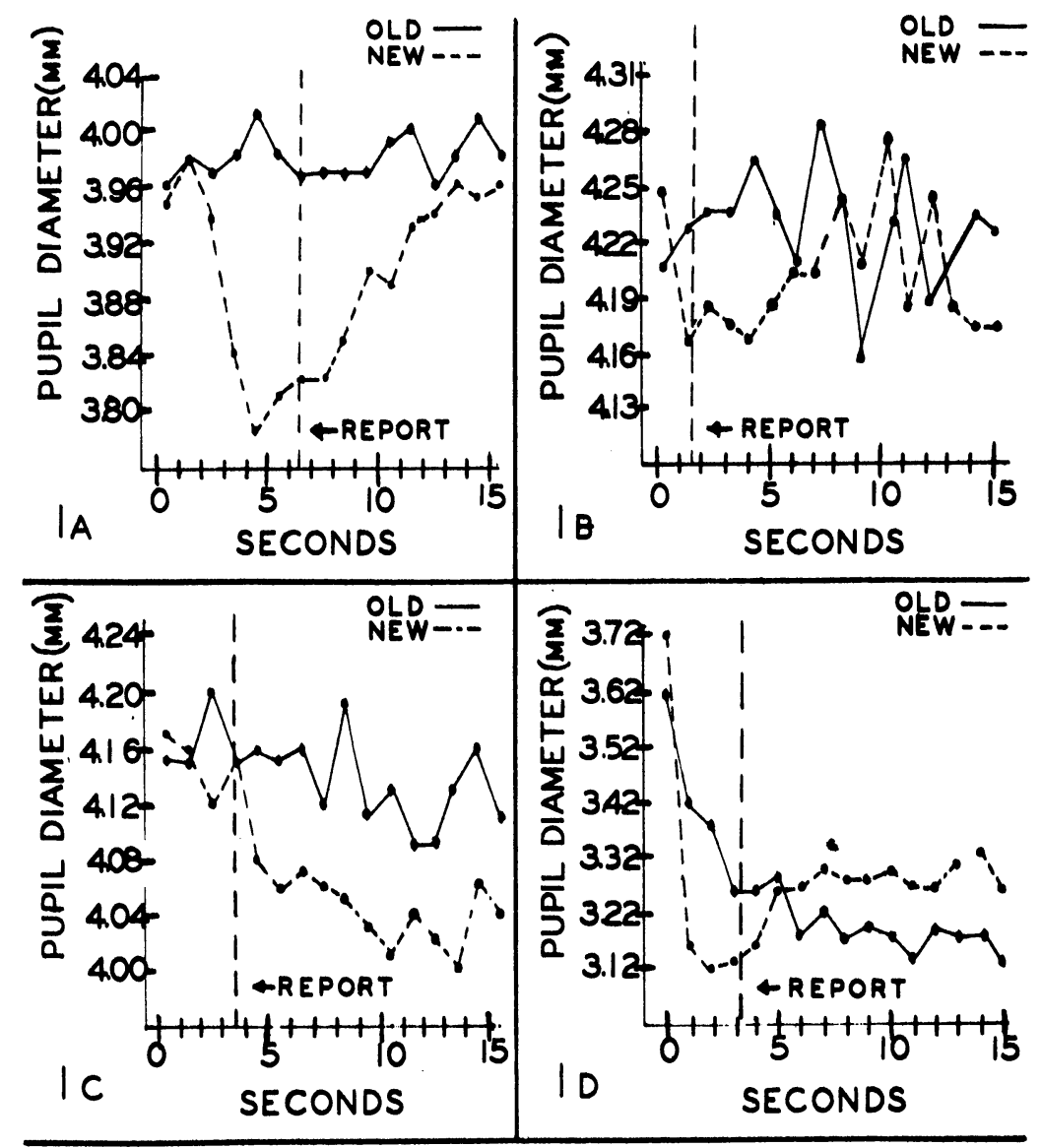

Fig. 1. Pupil diameters in millimeters with respect to reaction latency.

stimulus item and report was computed and is represented by a dashed vertical line in Fig. 1. Largest differences generally occur close to the report.

\section{DISCUSSION}

For all Ss, the predominant mode of pupillary reaction to stimulus novelty was not shown to be in the direction of dilation but in the opposite direction. That is, it can be inferred that stimulus novelty brought about inhibition of the pupillary orienting reflex with respect to well-formed recognition memory. This observation is in contradiction with Sokolov's theory, which usually predicts elicitation of the orienting reflex by stimulus novelty in a similar situation. The relation between the mechanisms of orienting reflex and memory is an inductive one.

Outside the Pavlovian framework, studies of pupillary reaction with respect to memory have predominantly addressed them selves to pupillary dilation, and the possibility of pupillary reaction in the opposite direction originating from some state of memory has not been investigated. A study by Beatty \& Kahneman (1963), for example, has shown that pupillary dilation occurs to telephone numbers stored in both short-term and long-term memory with somewhat greater dilation occuring in the latter. Unfortunately, the amount of dilation attributable to memory load is intermixed with pupillary changes occasioned by decision factors alone. That is, some pupil dilation may occur as a result of searching memory for item, regardless of whether the item is indeed stored in memory and well maintained. The question which was not raised was whether differential pupillary reactions occur to an item which has been learned and stored in memory as compared to. an item that has not been learned. On the other hand, this same question constitutes a crucial issue in deciding whether an inductive relation does exist between the mechanisms of the orienting reflex and conditioned reflex in particular, and between the mechanisms of attentional and memory mode of behavior in general. Inhibition of pupillary orienting reflex by stimulus novelty as demonstrated supports the original theory of Pavlov's concerning the nature of negative induction as applied to orienting reflex.

\section{REFERENCES}

Archer J. E. A re-evaluation of the meaningfulness of all possible CVC trigrams. Psychological Monographs, 1960, 74(Whole No. 10).

Beatty, J., \& Kahneman, D. Pupillary changes in two memory tasks. Psychonomic Science, 1966, 5, 371-372.

Hess, E. Attitude and pupil size. Scientific American, 1965, 212, 46-54.

Pavlov, I. P. Conditioned reflex. Oxford, England: Oxford University Press, 1927 (Dover, 1960).

Pavlov, I. P. Lectures on conditioned reflex. New York: International Publisher, 1928.

Sokolov, Ye. N. Perception and the conditioned reflex. New York: Pergamon, 1963.

(Received for publication November 5, 1973.) 